\title{
Sostdc1 regulates natural killer cell maturation and cytotoxicity
}

Alberto J. Millan ${ }^{1}$, Sonny R. Elizaldi ${ }^{1}$, Eric M. Lee ${ }^{1}$, Jeffrey O. Aceves ${ }^{1}$, Deepa

Murugesh ${ }^{2}$, Gabriela G. Loots ${ }^{1},{ }^{2}$, and Jennifer O. Manilay ${ }^{1}$

${ }^{1}$ University of California - Merced, Department of Molecular Cell Biology, School of Natural Sciences, Merced, CA, 95343, USA

${ }^{2}$ Lawrence Livermore National Laboratories, Physical and Life Sciences Directorate, Livermore, CA 94550, USA.

Corresponding Author:

Jennifer O. Manilay, PhD

University of California - Merced

School of Natural Sciences

5200 North Lake Road

Merced, CA 95343

Telephone: 209-228-4175

Email: jmanilay@ucmerced.edu 


\section{Abstract}

Natural killer (NK) cells are specialized lymphocytes with the innate ability to eliminate virally infected and cancerous cells, but the mechanisms that control NK cell development and cytotoxicity are incompletely understood. We identified novel roles for Sclerostin domain containing-1 (Sostdc1) in NK cell development and function. Sostdc1knockout (Sostdc1 ${ }^{-/}$) mice display a progressive accumulation of transitional NK cells $\left(\mathrm{CD} 27^{+} \mathrm{CD} 11 \mathrm{~b}^{+}, \mathrm{tNK}\right)$ with age, indicating a partial developmental block. The Ly49 repertoire on NK cells in Sostdc1 $1^{-/-}$mice is also changed. Lower frequencies of Sostdc1/- splenic tNKs express inhibitory Ly49G2 receptors, but higher frequencies express

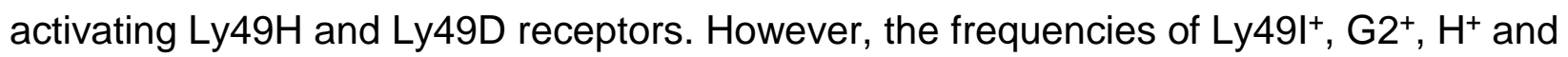
$\mathrm{D}^{+}$populations were universally decreased at the most mature (CD27-CD11 $\mathrm{b}^{+}, \mathrm{mNK}$ ) stage. We hypothesized that the Ly49 repertoire in Sostdc1 $1^{-/-}$mice would correlate with NK killing ability, and observed that Sostdc1 ${ }^{-/}$NK cells are hyporesponsive against MHC-I-deficient cell targets in vitro and in vivo, despite higher CD107a surface levels and similar IFN $\gamma$ expression to controls. Consistent with Sostdc1's known role in the regulation of Wnt signaling, high levels of Wnt coactivators Tcf7 and Lef1 were observed in Sostdc1 ${ }^{-/}$NK cells. Expression of the NK development gene Id2 was decreased in Sostdc1\% iNK and tNK cells, but we observed no changes in Eomes and Tbx21 expression. Reciprocal bone marrow transplant experiments showed that Sostdc1 regulates NK cell maturation and expression of Ly49 receptors in a cellextrinsic fashion from both non-hematopoietic and hematopoietic sources. Taken together, these data support a role for Sostdc1 in the regulation of NK cell maturation, and NK cell cytotoxicity, and identify potential NK cell niches. 


\section{Summary of Results}

- Sostdc1 ${ }^{-1-}$ mice display a partial block between the tNK and mNK developmental stages.

- Sostdc1 influences the Ly49 receptor repertoire on NK cells.

- NK cells in Sostdc1 ${ }^{-- \text {mice }}$ display impaired ability to kill $\beta_{2} \mathrm{~m}^{-/-}$target cells.

- Sostdc1 $1^{-1-}$ NK cell subsets express high levels of Wnt coactivators Tcf7 and Lef1.

- Id2 expression is decreased in iNK and tNK cells in the absence of Sostdc1.

- Bone marrow transplantation experiments demonstrate cell-extrinsic regulation of NK cell maturation by Sostdc1 in both non-hematopoietic (stromal) and hematopoietic cells. 


\section{Background and Rationale}

Natural Killer (NK) cells are innate lymphocytes that are important for early

3 immune defense against tumors and virally infected cells. Since the initial discovery of

4 NK cells in 1975, studies from many groups have identified NK cell receptors that are

5 involved in self/non-self recognition, NK cell precursors and stages of maturation,

6 cytokines and transcription factors that are critical for NK cell development and function,

7 and evidence for NK cell immune memory ${ }^{1-9}$. Despite over 40 years of NK cell history,

8 the molecular and cellular mechanisms that drive and integrate these processes is still

9 unclear. In particular, how the microenvironment regulates NK cell maturation and

10 function is still an area of ongoing investigation.

11 Conventional NK cells develop in the bone marrow (BM) from hematopoietic

12 stem cells (HSCs) following a well-established sequence of maturational stages and

13 egress to the peripheral organs to fully mature and function ${ }^{10,11,12}$. NK cell maturation

14 (Figure 1A) originates with the immature NK (CD27+CD11b;, iNK) cells, which

15 progresses to the transitional NK cell stage $\left(C D 27^{+} \mathrm{CD} 11 \mathrm{~b}^{+}, \mathrm{tNK}\right)$, then to the final

16 mature stage $\left(\mathrm{CD}^{2} 7^{-} \mathrm{CD} 11 \mathrm{~b}^{+}, \mathrm{mNK}\right)^{13-16}$. As $\mathrm{NK}$ cells progress through these stages,

17 they lose proliferative and cytokine-producing capability, but gain cytotoxic ability

18 against target cells ${ }^{12,17,18}$. Although the BM microenvironment is critical for NK cell

19 development, how the peripheral microenvironment regulates NK cell maturation and

20 cytotoxicity is incompletely understood and requires further investigation.

21 Sclerostin domain containing-1 (Sostdc1), also known as Wise, Ectodin, Usag-1,

22 and Sost-like, has been studied in the context of tooth development, kidney disease,

23 hair follicle formation, and bone fracture ${ }^{19-25}$. Sostdc1 can function as an antagonist of 
24 both Bone Morphogenetic Protein (BMP) and canonical Wnt signaling pathways ${ }^{20,21,23}$.

25 Sostdc1 expression is highly expressed in skin, brain, and intestine, as well as in

26 skeletal muscles, kidney, lungs, and vasculature $20,22,23$. Most recently, we found it also

27 to be expressed in the bone periosteum and mesenchymal stem cells (MSCs) to

28 support bone formation and fracture remodeling ${ }^{26}$. Here, we reveal Sostdc1's cell-

29 extrinsic roles in the regulation of NK cell maturation, Ly49 receptor expression and

30 cytotoxic function in the BM and spleen. 


\section{Results}

32 Sostdc1 ${ }^{-1-}$ mice display a partial block at the tNK stage

Our previous studies demonstrated that femurs of Sostdc1 $1^{-/-}$mice display a 21

34 percent increase in BM cavity volume compared to WT controls ${ }^{26}$. Consistent with this,

35 the total BM cellularity of Sostdc1 $1^{-/-}$bones was increased (Figure 1B). Sostdc1 $1^{-/-}$mice

36 also displayed higher total splenic cell numbers (Figure 1C). The increased cellularity

37 suggested that the Sostdc1 $1^{-/-} \mathrm{BM}$ and spleen microenvironments may be altered, and

38 that immune cell development may also be affected by the loss of Sostdc1. To test this,

39 we performed flow cytometry (FCM) and observed no differences in frequencies or

40 absolute numbers of $\mathrm{CD} 19^{+} \mathrm{B}$ lymphocytes, $\mathrm{CD} 3^{+} \mathrm{T}$ lymphocytes, and CD11 $\mathrm{b}^{+} \mathrm{Gr}^{+}$

41 granulocytes (data not shown). However, the frequency of CD11b+ Gr1- cells in the

42 Sostdc1 $1^{-/}$spleen was reduced. To determine if the CD11 ${ }^{+}$Gr1- cells were monocytes

43 or NK cells, we performed more detailed analysis with anti-NK1.1. Total NK (Live, CD3-

44 ,CD19-,Gr1',NK1.1+) frequencies were not affected, but total NK cell numbers were

45 increased only in the BM of Sostdc1/- mice (Figure 1D-1G). We then investigated if lack

46 of Sostdc1 affected NK cell maturation (Figure 1A), and discovered that Sostdc1 $1^{-/-}$mice

47 exhibit a partial block between the tNK (NK1.1 $\left.1^{+} \mathrm{CD} 11 \mathrm{~b}^{+} \mathrm{CD} 27^{+}\right)$and $\mathrm{mNK}\left(\mathrm{NK} 1.1^{+}\right.$

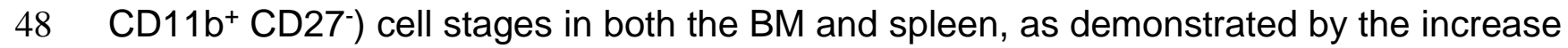

49 in frequency and number of tNK cells in the BM (Figures $\mathbf{1 H}$ and $\mathbf{1 I}$ ) and spleen

50 (Figures $\mathbf{1 J}$ and $\mathbf{1 K}$ ), and the decreased frequency of $\mathrm{mNKs}$ in the spleen. These data

51 indicated that Sostdc1 is required for full developmental progression from the tNK to the

$52 \mathrm{mNK}$ cell stages. 
Absence of Sostdc1 alters the Ly49 receptor repertoire on NK cells

We also examined if $\mathrm{NK}$ cells in Sostdc1 ${ }^{-/}$mice expressed different levels and distributions of inhibitory (Ly49I and Ly49G2) and activating (Ly49D and Ly49H) Ly49

57 receptors. FCM analysis of the Ly49 repertoire on iNKs, tNKs, and $\mathrm{mNKs}$ in Sostdc1 $1^{-/}$

58 mice revealed decreased frequencies of $L y 49 G 2^{+}$cells at all NK cell stages in the BM

59 (Supplemental Figure 1A-1C) and spleen (Figure 1L-1N). In contrast, frequencies of

$60 \mathrm{Ly}_{49 \mathrm{H}^{+}}$iNK and tNK cells in the Sostdc1/- BM and spleen were higher than controls, but

61 the frequencies of $\mathrm{Ly}_{4} 4 \mathrm{H}^{+} \mathrm{mNK}$ cells were reduced in both tissues (although only

62 statistically significant in the spleen). Similarly, frequencies of Ly49D ${ }^{+}$in iNK and tNK

63 cells were increased and reduced amongst the mNKs in the Sostdc1\% BM and spleen

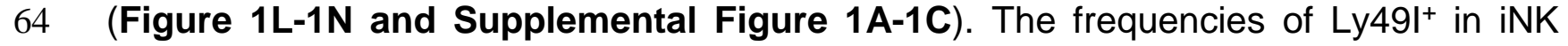

65 and tNK cells were similar to controls, but frequencies of $\mathrm{Ly}_{49 \mathrm{I}^{+}} \mathrm{mNK}$ cells were

66 decreased in Sostdc1 $1^{-/-}$mice (Figure $\mathbf{1 N}$ and Supplemental Figure 1C). The median

67 fluorescent intensity (MFI) of staining for Ly49G2 and Ly49H was reduced on Sostdc1 $1^{-/-}$

68 BM mNK cells only, indicating a relatively minor effect of Sostdc1 on cell surface Ly49

69 receptor expression levels (Supplemental Figure 1D-1K).

Since it is theorized that NK cell activity is governed by the combined set of Ly49

71 receptors expressed on a given NK cell, we further compared the frequencies of WT

72 and Sostdc1-- NK cells that express different combinations of Ly49 receptors ${ }^{\mathbf{2 7}}$

73 (Supplemental Figure 2). Higher frequencies of iNK and tNK cells expressing more

74 activating than inhibitory receptors (i.e. "activating repertoires") were observed in

75 Sostdc1 ${ }^{-/}$mice (Supplemental Figure 2D and 2E). However, lower frequencies of 
$76 \mathrm{mNK}$ cells with activating repertoires were observed (Supplemental Figure 2F). Taken

77 together, these data show the lack of Sostdc1 influences the Ly49 receptor repertoire.

NK cells in Sostdc1 $1^{-1-}$ mice are impaired in their ability to kill $\beta_{2} m$-deficient targets

The reduced frequency of splenic mNK with "activating repertoires" that favored

81 activation suggested that NK cell cytotoxicity in Sostdc1 ${ }^{-/-}$mice would be impaired. To

82 determine if the alterations in NK Ly49 repertoire correlated with NK cell killing ability,

83 we analyzed Sostdc1 $1^{-/}$NK cell cytotoxicity with novel FCM-based in vivo and in vitro

84 killing assays (Figure 2 and Supplemental Figure 3A-3E). Beta-2 microglobulin

85 knockout $\left(\beta_{2} \mathrm{~m}^{-/-}\right)$cells express little to no cell surface class I major histocompatibility

86 complex $(\mathrm{MHC} \mathrm{I})$ molecules and therefore are sensitive targets for $\mathrm{NK}$ cell killing ${ }^{28}$. To

87 test NK cell killing in vivo, we pre-activated NK cells in Sostdc1 ${ }^{-/-}$and WT control mice

88 with poly $(\mathrm{I}: \mathrm{C})^{29}$ (Figure 2A) and challenged them with equal numbers of $\beta_{2} \mathrm{~m}^{-/-}$and beta-

892 microglobulin-sufficient $\left(\beta_{2} \mathrm{~m}^{+/+}\right)$target cells, each labeled with two different

90 fluorochromes (Figure 2B). $\beta_{2} \mathrm{~m}^{+/+}$target cells from WT and Sostdc1-- mice were both

91 included as negative "self" controls (Figure 2C-2E and data not shown). After 14

92 hours of target cell challenge, we quantified the remaining $\beta_{2} \mathrm{~m}^{-/-}$, WT $\left(\beta_{2} \mathrm{~m}^{+/+}\right)$and

93 Sostdc1 $1^{-/}\left(\beta_{2} \mathrm{~m}^{+/+}\right)$targets by $\mathrm{FCM}$, to determine the frequency of live cells in each target

94 population (Figure 2A, 2C and 2D), and calculated the ratio of WT $\left(\beta_{2} \mathrm{~m}^{+/+}\right)$, Sostdc1 $1^{-/-}$

$95\left(\beta_{2} \mathrm{~m}^{+/+}\right)$, and $\beta_{2} \mathrm{~m}^{-/-}$targets in each setting (Figure 2E). An increased proportion of $\beta_{2} \mathrm{~m}^{-}$

$96{ }^{I-}$ targets remained in the Sostdc $1^{-/}$mice compared to WT controls (Figure 2E). We

97 confirmed that there was no effect on fluorophore labeling on $\beta_{2} \mathrm{~m}^{-/-}$cell target killing

98 with reciprocal labeling of targets of opposing fluorophore (Figure 2C and 2D). Thus, 
99 these results demonstrated that NK cells in Sostdc1 1 - mice have impaired killing ability

100 (Figure 2E).

This result was confirmed using an in vitro NK cell killing assay (Supplemental

102 Figure 3) using enriched NK cells from Sostdc1 ${ }^{-/}$and WT mice ${ }^{30}$, challenged with

103 fluorescently labeled $\beta_{2} \mathrm{~m}^{-/-}$or $\beta_{2} \mathrm{~m}^{+/+}$targets for 4 hours, in effector to target (E:T) ratios

104 of 1:1, 2:1, and 4:1 (Supplemental Figure 3A-3C). As expected, WT NK and Sostdc1/-

105 NK cells did not lyse $\beta_{2} \mathrm{~m}^{+/+}$targets at any $\mathrm{E}: \mathrm{T}$ ratio (Supplemental Figure 3D).

106 However, as shown in Supplemental Figure 3E, Sostdc1 ${ }^{--}$NK cells have reduced 107 capacity to lyse $\beta_{2} \mathrm{~m}^{-/-}$targets, even at the highest $4: 1 \mathrm{E}: \mathrm{T}$ ratio, indicating their 108 hyporesponsiveness to $\beta_{2} \mathrm{~m}^{-/-}$targets. FCM analysis of Sostdc1 ${ }^{-/} \mathrm{NK}$ cells to produce 109 the cytokine IFN $\gamma$ after stimulation revealed comparable levels to WT controls 110 (Supplemental Figure 3F and 3G). Surprisingly, activated Sostdc1 ${ }^{-/}$NK cells at all 111 developmental stages expressed significantly increased levels of the degranulation

112 marker CD107a (Supplemental Figure 3H and 31). Taken together, these results 113 suggest that Sostdc1 ${ }^{-/}$NK cell cytotoxicity is impaired, despite their ability to produce 114 comparable levels of IFN $\gamma$ and evidence of elevated accumulation of cytotoxic granules 115 at the cell surface.

117 Sostdc1-KO NK cell subsets upregulate Wnt genes Tcf7 and Lef1

118 Given that Sostdc1 is a known antagonist to canonical Wnt signaling, we 119 hypothesized that expression of canonical Wnt pathway transcription factors would be 120 increased in Sostdc1 ${ }^{--}$NK cell subsets. We purified iNK, tNK, and mNK cells by FCM 121 and analyzed expression of Wnt pathway genes $T c f 7^{3,31}$, Lef1 ${ }^{32}$, Axin2 ${ }^{33}$ and $M y c^{34}$, by 
122 real-time quantitative polymerase chain reaction (qPCR). Our results showed that

123 relative to WT subsets, Sostdc1 $1^{-/}$splenic tNK and mNK cells express significantly higher

124 levels of Tcf7, and tNK cells also show significantly increased expression of Lef1

125 (Figure 3A), consistent our hypothesis. Alternatively, we did not observe an increase in 126 Axin2 and Myc expression in any Sostdc1 ${ }^{-/}$NK cell subsets (Figure 3A). Together, 127 these results support a role for Wnt signaling by Tcf7 and Lef1 in NK cells of Sostdc1 ${ }^{-/-}$ 128 mice.

We also analyzed expression of transcription factors that govern NK cell 130 maturation. T-box family members, Eomesodermin (Eomes) and T-box protein 21 131 (Tbx21), have been shown to play a crucial role in early immature and mature NK cell 132 maturation ${ }^{35,36}$. Additionally, Eomes-deficient NK cells have reduced Ly49A, Ly49D, 133 Ly49G2, and Ly49H frequencies ${ }^{35}$. Inhibitor of DNA-binding 2 (Id2) is an early TF 134 involved in NK and innate lymphoid cell (ILC) lineage commitment ${ }^{36,37,38}$. Id2-deficient 135 mice have fewer mature NK cells and impaired cell killing in vitro ${ }^{39}$. Since Sostdc1 ${ }^{-/}$ 136 mice display a partial maturation block at the tNK cell stage and altered Ly49 receptor

137 frequencies (Figure 1), we hypothesized that we would observe decreased expression 138 of Eomes, Tbx21, and Id2 at distinct NK cell stages ${ }^{35-42}$. We found Id2 expression was

139 decreased in iNK and tNK cells in Sostdc1 ${ }^{-/}$mice (Figure 3B). These results suggest a 140 strong regulation of Id2 by Sostdc1 at early NK cell stages, whereas Eomes and Tbx21 141 are not regulated by Sostdc1. 
To determine if and how Sostdc1 within specific microenvironmental cell types

145 contributes to the partial block in NK cell maturation and changes in Ly49 repertoires,

146 we performed whole bone marrow transplantation (BMT) experiments. In order to

147 investigate if Sostdc1 in non-hematopoietic cells influenced NK cell development, we

148 first transplanted whole bone marrow cells from WT(CD45.1 $\left.{ }^{+} / 5.1^{+}\right)$donors into lethally-

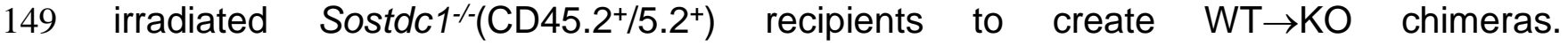

150 WT(CD45.1 $\left.1^{+} / 5.1^{+}\right) \rightarrow \mathrm{WT}\left(\mathrm{CD} 45.2^{+} / 5.2^{+}\right)$control chimeras were also prepared (Figure

151 4A). Fourteen weeks post-BMT, we analyzed donor-derived NK cell subsets and Ly49

152 receptor frequencies by FCM. Splenic NK cell numbers were increased (Figure 4B),

153 and $\mathrm{WT} \rightarrow \mathrm{KO}$ chimeras displayed a partial block between tNK and mNK cell stages in

154 the spleen (Figure 4C-4E) and BM (Supplemental Figure 4C-4E), similar to the 155 phenotype that was observed in the non-transplanted Sostdc1 ${ }^{\%}$ mice (Figure $\mathbf{1 H - 1 K}$ ).

$156 \mathrm{Ly}_{49 \mathrm{H}^{+}} \mathrm{mNK}$ cells were decreased in WT $\rightarrow \mathrm{KO}$, similar to non-transplanted Sostdc1 ${ }^{-/}$

157 mice in the spleen (Figure 4F and Figure 1N). In contrast, WT $\rightarrow$ KO chimeras

158 contained decreased frequencies of splenic $\mathrm{Ly}_{49 \mathrm{H}^{+}}$iNK and tNK cells compared to

$159 \mathrm{WT} \rightarrow \mathrm{WT}$ controls, a result that was the opposite of the increased frequencies of $\mathrm{Ly}_{4} 4 \mathrm{H}^{+}$

160 cells within these NK subsets of non-transplanted Sostdc1 ${ }^{-/}$mice (Figure 4F and

161 Figure 1L-1M). In addition, no differences in Ly49G2 and Ly49D subsets were 162 observed between the $\mathrm{WT} \rightarrow \mathrm{KO}$ and control chimeras, a result that also differed from

163 the non-transplanted Sostdc1 ${ }^{-/}$mice in the spleen (Figure 4F and Figure 1L-1N) and 164 BM (Supplemental Figure 4F). Taken together, these results suggested that Sostdc1

165 in non-hematopoietic cells controls progression from tNK to mNK stages and NK 166 cellularity, but plays a smaller role in shaping the Ly49 receptor repertoire. 
Sostdc1 in a hematopoietic cell lineage other than NK cells regulates the Ly49 receptor

168 repertoire

We next prepared reciprocal KO $\rightarrow$ WT chimeras (whole BM cells from Sostdc1 ${ }^{-}$ $\left(C D 45.2^{+} / 5.2^{+}\right)$donors transplanted into lethally irradiated WT $\left(C D 45.1^{+} / 5.1^{+}\right)$recipient mice) to determine how Sostdc1 ${ }^{-1}$ NK cells mature and if their Ly49 receptor frequency was changed in a Sostdc1-sufficient microenvironment (Figure 5A). Remarkably, splenic NK cell numbers (Figure 5B) and maturation (Figure 5C-5E) were not affected in $\mathrm{KO} \rightarrow \mathrm{WT}$ chimeras, in contrast to the non-transplanted Sostdc1 ${ }^{-1}$ mice and the WT $\rightarrow$ KO chimeras (Figure $\mathbf{1 H}-\mathbf{1 I}$ and Figure 4B-4E, respectively). However, the BM analysis showed an increase in NK cell numbers (Supplemental Figure $\mathbf{4 H}$ ) and a similar tNK cell accumulation as observed in the non-transplanted Sostdc $1^{-/}$mice (Figure 1H-1K and Supplemental Figure 4K). Furthermore, analysis of donor-derived Ly49-expressing NK cell subsets in the spleens in KO $\rightarrow$ WT chimeras demonstrated some similar patterns as non-transplanted Sostdc1 $1^{-1}$ mice, such as the increase in frequencies of $\mathrm{Ly}_{4} 4 \mathrm{H}^{+}$and $\mathrm{Ly} 49 \mathrm{D}^{+} \mathrm{tNK}$ cells and a decrease in the frequencies of Ly49G2 ${ }^{+}$iNK cells, and decreased frequencies of $\mathrm{Ly}_{49 G 2^{+}}$and $\mathrm{Ly}_{49 I^{+}} \mathrm{mNK}$ cells (Figure 5F and Figure 1L-1N). However, higher frequencies of mNK cells expressing Ly49H and Ly49D were observed in the $\mathrm{KO} \rightarrow \mathrm{WT}$ spleens, whereas these populations were decreased in non-transplanted Sostdc1 ${ }^{-/}$mice (Figure 5F and Figure $1 \mathrm{~L}-\mathbf{1 N}$ ).

The Ly49 frequency patterns observed in the $\mathrm{KO} \rightarrow \mathrm{WT}$ chimeras strongly suggested that Sostdc1 in NK cells regulated the Ly49 repertoire in a cell-intrinsic fashion. To confirm this, we examined Sostdc1 expression in sorted WT iNK, tNK, and mNK cells by qPCR. Surprisingly, iNK, tNK, and mNK cells do not express Sostdc1 
190 (Figure 5G). To determine alternative possible hematopoietic sources of Sostdc1, we 191 examined other populations, such as Lineage-Sca1 ${ }^{+} \mathrm{Kit}^{+}$(LSK), Lineage-Kit ${ }^{+}$Sca1- $($LK), 192 and common lymphoid progenitors (CLP), NK T cells, macrophages, B cells and 193 granulocytes, which were all negative for Sostdc1 (Figure 5G). Only CD4 ${ }^{+}$and CD8 ${ }^{+} \mathrm{T}$ 194 cells displayed high levels of Sostdc1 expression (Figure 5G). This expression pattern 195 was confirmed in CD4 and CD8 T cells from Sostdc1 ${ }^{-1-}$ mice using PCR for LacZ ${ }^{26}$ (data 196 not shown). Collectively, these results indicate that Sostdc1 does not regulate splenic 197 NK cell development in a NK cell-intrinsic manner, and identifies Sostdc1-positive T 198 cells as putative NK niche cells that may contribute to shaping of the Ly49 repertoire. 


\section{Discussion}

We have uncovered novel roles of the Sostdc1 gene in NK cell maturation and

200 function through two distinct mechanisms. Our working model is illustrated in Figure 6.

201 Our data supports that Sostdc1 from two distinct sources, non-hematopoietic stromal

202 cells, and hematopoietic cells (in particular, CD4 ${ }^{+}$and $\mathrm{CD}^{+} \mathrm{T}$ lymphocytes) regulate NK

203 cell maturation versus Ly49 receptor expression and frequencies, respectively, in

204 somewhat independent manners, and that this occurs through the control of Wnt

205 signaling activation. Our developmental and functional NK cytotoxicity assay results

206 lead us to conclude several NK "niche cell" populations exist that require Sostdc1

207 expression in order to produce a healthy NK cell repertoire that can distinguish between

208 self and non-self.

209

Perhaps the most surprising finding from our studies is that Sostdc1 is not

210 expressed in NK cells themselves, and that Ly49 receptor expression in the spleen is

211 possibly controlled in a cell-extrinsic manner by $\mathrm{CD}^{+}$and $\mathrm{CD}^{+} \mathrm{T}$ cells. To our

212 knowledge, there is no previous demonstration of NK and T cell crosstalk that shapes

213 the NK Ly49 receptor profile. Recent studies have defined Sostdc1 as a marker of

214 relatively rare memory $\mathrm{PD}-1^{+} \mathrm{CD} 4^{+} \mathrm{T}$ cells $\mathrm{s}^{43,44}$ and $\mathrm{T}$ follicular helper $\left(\mathrm{T}_{\mathrm{FH}}\right)$ cells found in

215 the Peyer's Patches and peripheral lymph nodes ${ }^{10}$, but only cite Sostdc1's role on

216 humoral immunity. We observed high expression of Sostdc1 in bulk sorted splenic

$217 \mathrm{CD}^{+}$and $\mathrm{CD}^{+}$cells from the spleen, and we assume that most of the Sostdc1

218 expression is coming from $\mathrm{PD}^{+} \mathrm{CD}^{+} \mathrm{T}$ cells and $\mathrm{T}_{\mathrm{FH}}$ cell subsets, based on these

219 published studies. We have not observed any obvious block in T cell development in

220 the Sostdc1/- mice (data not shown). Undeniably, further experimentation is required to 
221 definitively connect the role of these $\mathrm{T}$ cell subsets in NK cell regulation, and to

222 determine whether the $\mathrm{T}$ cells are regulating Ly49 receptor expression via directly

223 binding to NK cells, or mediating their effects indirectly by secreting Sostdc1 in a 224 paracrine fashion.

Wnt signaling has been well studied in the framework of hematopoietic stem cells (HSCs) to help promote proliferation, differentiation, and homeostasis ${ }^{45,46}$. It is now evident that canonical Wnt signaling plays a crucial role in the regulation of many 228 immune cells ${ }^{45}$. Based on the antagonist role of Sostdc1 on Wnt signaling and our 229 discovery of increased Tcf7 and Lef1 expression in NK cells from Sostdc1\% mice, we 230 conclude that canonical Wnt signaling plays a crucial role in NK cell development and 231 function, particularly at the tNK cell stage. In the absence of Sostdc1, tNK cells are 232 partially blocked in their maturation, express dysregulated Ly49 frequencies in the BM 233 and spleen, and appear to be more reliant on Wnt activation. Our observations that one 234 of the coactivators of canonical Wnt signaling, Tcf7, was significantly upregulated in tNK 235 and mNK cells, and the secondary coactivator, Lef1, was significantly upregulated in the $236 \mathrm{mNK}$ cell stage, suggest that a critical period exists in which tNK cells require 237 downregulation of Tcf7 and Lef1 in order to progress to the mNK cell stage. Our results 238 and interpretation are consistent with a recent study that downregulation of Tcf1 239 (encoded by Tcf7) is required for full NK cell maturation and cytotoxicity ${ }^{3}$.

240 Based on our current findings, we cannot rule out whether the impaired killing of $241 \beta_{2} \mathrm{~m}^{-/-}$targets by NK cells from Sostdc1 $1^{-/-}$mice is due to insufficient numbers of $\mathrm{mNK}$ 242 cells, inefficient execution of the perforin and granzyme pathways, the dysregulation of 243 Ly49 receptor frequencies amongst NK cells, or a combination of all of these possible 
244 mechanisms. NK cells at tNK and mNK stages express genes involved in cytotoxic

245 function ${ }^{15,11,12}$. It has been shown that Ly49 receptor expression is required for NK cell

246 cytoxicity ${ }^{47}$, which is consistent with our observations that the splenic mNK cells in the

247 Sostdc1 $1^{-/-}$mice contain decreased frequencies of all Ly49-expressing subsets and the

248 killing ability of Sostdc1 $1^{--}$NK cells towards $\beta_{2} \mathrm{~m}^{-/-}$targets is poor. Because Sostdc1 $1^{-/-}$

249 mice express increased proportions of tNK cells with an "activating" repertoire ${ }^{48}$ and

250 high CD107a levels, we would have expected enhanced target cell killing by the

251 Sostdc1 $1^{-/}$NK cells, but we observed them to be hyporesponsive. Taken together, our

252 results suggest that NK cell cytotoxicity is universally disabled in the absence of

253 Sostdc1, but is caused by distinct mechanisms in tNK and mNK cells. Further

254 experiments, in which the killing ability of purified tNK and mNK cells from Sostdc1 ${ }^{-/-}$

255 mice is specifically examined, are necessary to definitely demonstrate this. Additional

256 work is also needed to dissect the specific roles of non-hematopoietic and

257 hematopoietic cells on NK cell cytotoxicity. Understanding the details of the basic

258 biology underlying the development and regulation of NK cell cytotoxicity and how these

259 processes are quantitatively integrated could be applied to manipulate these processes

260 in a controlled fashion in order to produce specific numbers of NK cells with enhanced

261 killing ability, and perhaps impact the production of NK cell-based cancer

262 immunotherapies ${ }^{1-6}$. 


\section{Figure Legends}

264 Figure 1. Delayed NK cell development and altered Ly49 repertoire in Sostdc1 ${ }^{-/-}$

265 mice. A) Schematic diagram of NK cell development: NKP: NK progenitor (CD27266 CD11b-), iNK: immature NK (CD27 ${ }^{+}$CD11b-), tNK: transitional NK (CD27 ${ }^{+}$CD11b $\left.{ }^{+}\right)$, 267 mNK: mature NK (CD27- $\left.7^{-} D 11 b^{+}\right)$; B) Total cellularity of bone marrow (BM) in the 268 femurs and tibiae and C) spleen of wild-type (WT) and Sostdc1\% (KO) mice; D and F)

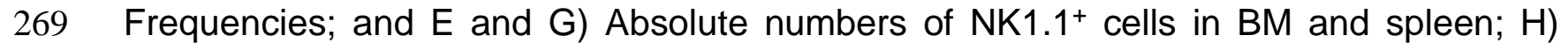
270 Representative flow cytometry plots showing NK cell stages in BM; I) Summary of 271 frequencies and absolute numbers of NKP, iNK, tNK and $\mathrm{mNK}$ cells in BM; J)

272 Representative flow cytometry plots showing NK cell stages in spleen; K) Summary of 273 frequencies and absolute numbers of NKP, iNK, tNK and mNK cells in spleen; L-N) 274 distribution of splenic NK cells expressing Ly49I, Ly49G2, Ly49H and Ly49D on iNK, 275 tNK and mNK cells. Asterisks indicate statistically significant differences between 276 means, as determined by Student's t-test. ${ }^{\star} p<0.05,{ }^{*} p<0.01,{ }^{\star *}{ }^{*} p<0.0001$. Each point 277 represents a single mouse.

279 Figure 2. Sostdc1 deficiency results in impaired NK cell killing. A) Scheme of in 280 vivo NK cell killing assay. B) Cartoon depicting how WT and $\beta_{2} \mathrm{~m}^{-/-}$target cells were 281 labeled for detection by FCM. C and D) Representative FCM plots showing distinct 282 populations of live WT and $\beta_{2} \mathrm{~m}^{-/-}$target in poly-I:C-treated WT and Sostdc1 ${ }^{-/-}$mice. The 283 left column shows representative FCM plots enumerating the frequencies of WT targets 284 in WT and Sostdc1-- mice. The middle and right column show representative FCM plots 285 enumerating the frequencies of reciprocally labeled $\beta_{2} \mathrm{~m}^{-/-}$and WT targets. E) Ratio of 
targets to show their distribution after 14 hours in vivo (Target 1 and Target 2 identified

287 in table below the graph). Each point represents an independent biological replicate.

$288{ }^{\star} p<0.05,{ }^{\star *} p<0.01$, Student's t-test.

289

290

Figure 3. Expression of Wnt pathway and NK cell development genes in Sostdc1/-

291 NK cell subsets. A) Gene expression of Wnt pathway genes Tcf7, Lef1, Axin2, and 292 Myc by quantitative PCR; B) Gene expression of NK cell development genes Eomes, 293 Id2 and Tbx21. Data in A and B are shown relative to WT controls. ${ }^{*} \mathrm{p}<0.05$, Student's 294 t-test.

295

296 Figure 4. Non-hematopoietic stromal cells regulate the maturation of NK cells. A)

297 Experimental scheme to create $\mathrm{WT} \rightarrow$ Sostdc1 $^{-/-}(\mathrm{WT} \rightarrow \mathrm{KO})$ bone marrow chimeras. B)

298 Total spleen cellularity (left) and total splenic NK cell numbers in chimeras (right); C)

299 representative FCM plots of NK cell maturation in donor (CD45.1 $1^{+}$)-derived NK cells in

300 WT $\rightarrow$ WT and WT $\rightarrow$ KO chimeras; D) quantification of donor-derived NK cell subset

301 frequencies and E) NK subset cellularity; F) analysis of Ly49 repertoire on donor302 derived iNK (left), tNK (center) and mNK (right) cells in WT $\rightarrow$ WT and WT $\rightarrow$ KO 303 chimeras. ${ }^{*} p<0.05{ }^{* \star} p<0.01,{ }^{\star \star *} p<0.001$, Student's t-test.

306 repertoire. A) Experimental scheme to create Sostdc $1^{-1-} \rightarrow \mathrm{WT}(\mathrm{KO} \rightarrow \mathrm{WT})$ bone marrow

307 chimeras. B) total cellularity in spleen (left) and total donor (CD45.2+)-derived NK cell

308 numbers (right) in chimeras; C) representative FCM plots of splenic NK cell maturation 
309 of donor-derived NK cells in WT $\rightarrow$ WT and KO $\rightarrow$ WT chimeras; D) quantification of donor

310 NK cell subset frequencies and E) NK cellularity in the spleen of chimeras; F) analysis

311 of Ly49 repertoire on donor-derived iNK (left), tNK (center) and mNK (right) cells in

$312 \mathrm{WT} \rightarrow \mathrm{WT}$ and $\mathrm{KO} \rightarrow \mathrm{WT}$ spleens. G) Sostdc1 expression in hematopoietic cell lineages

313 by RT-PCR. *p<0.05 **p<0.01, ${ }^{* \star *} p<0.001$, Student's t-test.

315 Figure 6. Working model of Sostdc1's role in NK cell maturation and cytotoxicity.

316 A) In a Sostdc1-sufficient (wild-type) microenvironment, Sostdc1 is expressed by non-

317 hematopoietic stromal cells in the bone that antagonizes Wnt signaling in NK cells, 318 resulting in baseline levels of Tcf7 and Lef1, and regulates NK cell numbers. Sostdc1 319 expressed by $\mathrm{T}$ cells also influences Wnt signaling genes, but distinctly controls the 320 distribution of the Ly49 repertoire. Collectively, Sostdc1 is required in the 321 microenvironment for development of NK cells with the ability to effectively recognize, 322 be primed for activation and lyse MHC-I deficient targets. B) In the absence of Sostdc1 323 in bone stromal cells, splenic stromal cells or T cells, Tcf7 and Lef1 expression is 324 increased as a result of overstimulated Wnt signaling, which adversely affects NK cell 325 numbers. Loss of Sostdc1 in T cells might also dysregulate the distribution of Ly49s 326 amongst NK cell subsets. Collectively, loss of Sostdc1 in stromal cells and T cells acts 327 cell-extrinsically on NK cells, producing NK cells that are hyporesponsive to MHC-I 328 deficient targets. 
Materials and Methods

Mice

Sostdc1 $1^{--}$mice have been described ${ }^{22,26}$, and both bred and transferred from

Lawrence Livermore National Laboratories (LLNL) to UC Merced to begin an

337 independent breeding colony. C57B6/J (CD45.2 $\left./ 5.2^{+}\right)$and B6.SJL-Ptprca Pepcb/BoyJ

$338\left(\mathrm{CD} 45.1^{+} / 5.1^{+}\right)$and B6.129P2-B2mtm1Unc/J $\left(\beta_{2} \mathrm{~m}^{-/}\right)$mice were obtained from Jackson

339 Laboratories. Mice of 28-38 weeks of age and of both sexes were used. No differences

340 between sexes nor mice from LLNL and UC Merced colonies have been observed. All

341 mice were housed in conventional housing with autoclaved feed. Mice were euthanized

342 by carbon dioxide asphyxiation followed by cervical dislocation. All animal procedures

343 were approved by the UC Merced and LLNL Institutional Animal Care and Use

344 Committees.

Flow cytometry

Isolation of spleen and bone marrow cells were performed and stained for flow

348 cytometry as described ${ }^{26}$. Antibodies against CD161 or NK1.1 (PK136), CD11b (M1/70),

349 CD27 (LG.3A10), CD19 (6D5), CD3 (2C11), Gr1-Ly6C/G (Gr1), Ly49G2 (4D11), Ly49I

350 (YL1-90), Ly49H (3D10), Ly49D (eBio4E5), CD45.2 (104), CD45.1 (A20), CD45 (30-

351 F11), CD4 (GK1.5 ), CD8 (2.43), Ter119 (TER119), CD107a (1D4B), Rat IgG2a $\kappa$

352 Isotype Control (RTK2758), IFN $\gamma$ (XMG1.2), Rat IgG1 $\kappa$ Isotype control (RTK2071), and

353 BUV395 Streptavidin, eFluor 780 Fixable Viability Dye, eFluor 506 Fixable Viability Dye

354 were purchased from eBioscience, BioLegend, Miltenyi Biotec, and BD Biosciences.

355 Staining of all cells included a pre-incubation step with unconjugated anti-CD16/32 
356 (clone 2.4G2 or clone 93) mAb to prevent nonspecific binding of mAbs to Fc $\gamma \mathrm{R}$. For

357 extracellular staining, the cells were washed and incubated with a panel of mAbs for 15-

35820 minutes at $4 \mathrm{C}$ or on ice, and then washed again. Intracellular staining was

359 performed using the BD Cytofix/Cytoperm Fixation/Permeabilization Kit (BD

360 Biosciences) per the manufacturer's instructions. To purify NK cells and NK cell

361 subsets, enrichment of NK cells was first achieved by staining with biotinylated anti-

362 "lineage" cocktail (anti-CD3, CD4, CD8, CD19, Gr1, and Ter119) followed by magnetic

363 separation using EasySep Positive Selection kit (Stem Cell Technologies), After

364 enrichment, cells were stained with streptavidin-FITC, anti-CD27, and additional anti-

365 CD3, CD19, Gr1, CD45, NK1.1, and CD11b. Lineage-negative CD45+ NK1.1+ iNKs,

366 tNKs and mNKs were sorted on the FACS Aria II (Becton Dickinson). Flow cytometric

367 data was acquired on the BD LSR II or FACS Aria II cell sorter (Becton Dickinson). The

368 data was analyzed using Flowjo version 7.6 or 10 (Tree Star, Inc.)

\section{In vivo NK cell killing assay}

Sostdc1 $1^{-/-}$and B6 control mice received $200 \mu \mathrm{g}$ of polyinosinic-polycytidylic acid

372 (Poly(l:C)) (Sigma-Aldrich) via injection into the intraperitoneal cavity. Thirty-six hours

373 later, splenic cells from aged and sex-matched $\beta_{2} \mathrm{~m}^{-/-}$and $\beta_{2} \mathrm{~m}^{+/+}$(WT or Sostdc1 ${ }^{-/-}$) mice

374 were harvested and processed to a single cell suspension in media (Medium 199, 2\%

375 FCS, 2mM L-glutamine, 100U/ml penicillin, $100 \mu \mathrm{g} / \mathrm{ml}$ streptomycin, $25 \mathrm{mM}$ HEPES)

376 and counted using a hemocytometer. $\beta_{2} \mathrm{~m}^{+/+}\left(\mathrm{WT}\right.$ or Sostdc1 $\left.{ }^{-/-}\right)$and $\beta_{2} \mathrm{~m}^{-/-}$control target

377 cells were stained with anti-CD45 conjugated to either APC or PE in media for 20 min

378 on ice. $5 \times 10^{6} \beta_{2} \mathrm{~m}^{-/-}$stained splenic cells were mixed with $5 \times 10^{6} \beta_{2} \mathrm{~m}^{+/+}$stained control 
379 cells at a 50:50 ratio, thus providing a method to track each target cell type by flow

380 cytometry. Stained cell target cell mixtures were injected intravenously by retro-orbital

381 injection. Fourteen hours later, spleens from Sostdc1 ${ }^{-/-}$and WT recipients were then

382 harvested and processed for flow cytometry. NK cell lysis of targets was determined by

383 flow cytometry (FCM) and calculated by the ratio of live $\beta_{2} \mathrm{~m}^{+/+}(\mathrm{WT}), \beta_{2} \mathrm{~m}^{+/+}$(Sostdc1 $\left.1^{-/-}\right)$

384 and $\beta_{2} \mathrm{~m}^{-/-}$targets over WT control cells in the same mouse.

385

386 Gene expression analysis by quantitative PCR

387 Cells were pelleted and resuspended in RNeasy Lysis Buffer with 2388 mercaptoethanol (Qiagen). Total RNA was purified using Qiagen RNeasy Mini Kit 389 (Qiagen) according to manufacturer's protocol. RNA concentration and purity was 390 analyzed using a NanoDrop ND-1000 Spectrophotometer (Thermo Fisher Scientific). 391 iScript cDNA Synthesis Kit was used (Bio-Rad Laboratories) according to the 392 manufacturer's protocol. Real-time quantitative PCR performed using the iTaq Universal 393 SYBR Green Supermix kit (Bio-Rad Laboratories) and ran on a Stratagene Mx3000P 394 thermocycler (Thermo Fisher Scientific) using the following conditions: 1 cycle at $9^{\circ} \mathrm{C}$ for $39530 \mathrm{~s}$, followed by 40 cycles of $95^{\circ} \mathrm{C}$ for $5 \mathrm{~s}$ and $60^{\circ} \mathrm{C}$ for $30 \mathrm{~s}$, and a final cycle at $95^{\circ} \mathrm{C}$ 396 for $1 \mathrm{~min}, 55^{\circ} \mathrm{C}$ for $30 \mathrm{~s}$, and $95^{\circ} \mathrm{C}$ for $30 \mathrm{~s}$ to end the run. The PCR products were 397 visualized on a 2\% agarose gel and imaged under UV light using a ChemiDoc (Bio-Rad 398 Laboratories) with SYBR Safe (Invitrogen) stain. The list of genes and their primer 399 sequences are listed in Supplementary Table 1. 
Whole BM cells were aseptically isolated from B6 (CD45.1 or CD45.2) wild type

402 (WT) or Sostdc1 ${ }^{-1-}$ (CD45.2) mice and $5 \times 10^{6}$ cells were transferred via retro-orbital

403 injection into lethally (10 Gy) irradiated recipients 4 hours after irradiation using a

404 cesium irradiator. Mice were given neomycin-containing drinking water for 2 weeks

405 post-transfer. Chimeras were analyzed 14 weeks post-transplant.

406 


\section{Acknowledgments}

409 This work was supported by University of California (UC), Merced faculty research

410 funding, University of California Cancer Research Coordinating Committee grant and

411 Halcyon-Dixon Trust award to JOM, and UC Graduate Student Fellowships to AM.

412 GGL works under the auspices of the U.S. Department of Energy by Lawrence

413 Livermore National Laboratory under Contract DE-AC52-07NA27344. Authors' roles:

414 GGL and DM provided the Sostdc1\%- mice. Study design: AM and JOM. Study conduct:

415 AM, SE, EL, JA, JOM. Data collection: AM, SE, EL, JA. Data analysis: AM, SE, EL, JA,

416 JOM. Data interpretation: AM and JOM. Drafting manuscript: AM and JOM. Revising

417 manuscript content: AM and JOM. Approving final version of manuscript: JOM. JOM

418 takes responsibility for the integrity of the data analysis. The authors thank the staff of

419 the Department of Animal Research Services and the Flow Cytometry Core of the Stem

420 Cell Instrumentation Foundry at UC Merced for excellent animal care and technical

421 support, and for Dr. Anna Beaudin and Dr. Marcos E. García-Ojeda for their comments

422 on the manuscript. 


\section{References}

426

427

428

429

430

431

432

433

434

435

436

437

438

439

440

441

442

443

444

445

446

447

448

449

450

451

452

453

454

455

456

457

458

459

460

461

462

463

464

465

466

467

468

469

1. Cifaldi, L., Locatelli, F., Marasco, E., Moretta, L. \& Pistoia, V. Boosting Natural Killer Cell-Based Immunotherapy with Anticancer Drugs: a Perspective. Trends Mol Med 23, 1156-1175 (2017).

2. Geary, C.D. \& Sun, J.C. Memory responses of natural killer cells. Semin Immunol 31, 11-19 (2017).

3. Jeevan-Raj, B. et al. The Transcription Factor Tcf1 Contributes to Normal NK Cell Development and Function by Limiting the Expression of Granzymes. Cell Rep 20, 613-626 (2017).

4. Kim, S. et al. Licensing of natural killer cells by host major histocompatibility complex class I molecules. Nature 436, 709-713 (2005).

5. Lin, C. \& Zhang, J. Reformation in chimeric antigen receptor based cancer immunotherapy: Redirecting natural killer cell. Biochim Biophys Acta 1869, 200215 (2018).

6. Mehta, R.S., Randolph, B., Daher, M. \& Rezvani, K. NK cell therapy for hematologic malignancies. Int J Hematol 107, 262-270 (2018).

7. Manilay, J.O. \& Sykes, M. Natural killer cells and their role in graft rejection. Current Opinion in Immunology 10, 532-538 (1998).

8. Manilay JO, W.G., Sykes M. . Levels of Ly-49 receptor expression are determined by the frequency of interactions with MHC ligands: evidence against receptor calibration to a "useful" level. J Immunol 163, 2628-2633 (1999).

9. Manilay, J.O., Waneck, G.L. \& Sykes, M. Altered expression of Ly-49 receptors on NK cells developing in mixed allogeneic bone marrow chimeras. International Immunology 10, 1943-1955 (1998).

10. Claude, G. et al. The trafficking of natural killer cells. Immunological Reviews 220, 169-182 (2007).

11. Yokoyama, W.M., Kim, S. \& French, A.R. The dynamic life of natural killer cells. Annu Rev Immunol 22, 405-429 (2004).

12. Di Santo, J.P. Natural killer cell developmental pathways: a question of balance. Annu Rev Immunol 24, 257-286 (2006).

13. Huntington, N.D. et al. NK Cell Maturation and Peripheral Homeostasis Is Associated with KLRG1 Up-Regulation. The Journal of Immunology 178, 47644770 (2007). 
14. Hayakawa, Y. \& Smyth, M.J. CD27 Dissects Mature NK Cells into Two Subsets with Distinct Responsiveness and Migratory Capacity. The Journal of Immunology 176, 1517-1524 (2006).

15. Chiossone, L. et al. Maturation of mouse NK cells is a 4-stage developmental program. BLOOD 113 (2009).

16. Kim, S. et al. In vivo developmental stages in murine natural killer cell maturation. Nat Immunol 3, 523-528 (2002).

17. Orr, M.T. \& Lanier, L.L. Natural killer cell education and tolerance. Cell 142, 847856 (2010).

18. Sun, J.C. Re-educating natural killer cells. J Exp Med 207, 2049-2052 (2010).

19. Togo, Y. et al. Antagonistic Functions of USAG-1 and RUNX2 during Tooth Development. PLoS One 11, e0161067 (2016).

20. Ellies, D.L. et al. Wise regulates bone deposition through genetic interactions with Lrp5. PLoS One 9, e96257 (2014).

21. Kiso, H. et al. Interactions between BMP-7 and USAG-1 (uterine sensitizationassociated gene-1) regulate supernumerary organ formations. PLoS One 9, e96938 (2014).

22. Collette, N.M. et al. Sost and its paralog Sostdc1 coordinate digit number in a Gli3-dependent manner. Dev Biol 383, 90-105 (2013).

23. Tanaka, M. et al. Expression of BMP-7 and USAG-1 (a BMP antagonist) in kidney development and injury. Kidney Int 73, 181-191 (2008).

24. Saito, K. et al. Effects of Usag-1 and Bmp7 deficiencies on murine tooth morphogenesis. BMC Dev Biol 16, 14 (2016).

25. Collette, N.M., Genetos, D.C., Murugesh, D., Harland, R.M. \& Loots, G.G. Genetic evidence that SOST inhibits WNT signaling in the limb. Dev Biol 342, 169-179 (2010).

26. Collette, N.M. et al. Sostdc1 deficiency accelerates fracture healing by promoting the expansion of periosteal mesenchymal stem cells. Bone 88, 20-30 (2016).

27. Sternberg-Simon, M. et al. Natural killer cell inhibitory receptor expression in humans and mice: a closer look. Front Immunol 4, 65 (2013). 
28. Regner, M., Pavlinovic, L., Young, N. \& Mullbacher, A. In vivo elimination of MHC-I-deficient lymphocytes by activated natural killer cells is independent of granzymes A and B. PLoS One 6, e23252 (2011).

29. Alter, G., Malenfant, J.M. \& Altfeld, M. CD107a as a functional marker for the identification of natural killer cell activity. J Immunol Methods 294, 15-22 (2004).

30. Kehrl, J.H. et al. Novel interleukin 2 (IL-2) receptor appears to mediate IL-2induced activation of natural killer cells. J Clin Invest 81, 200-205 (1988).

31. Held, W., Clevers, H. \& Grosschedl, R. Redundant functions of TCF-1 and LEF-1 during T and NK cell development, but unique role of TCF-1 for Ly49 NK cell receptor acquisition. Eur J Immunol 33, 1393-1398 (2003).

32. Genderen, C.v. et al. Development of several oreans that require inductive epithelial-mesenchymal interactions is impaired in LEF-1- deficient mice. Genes \& Development 8, 2691-2703 (1994).

33. Jho, E.h. et al. Wnt/ -Catenin/Tcf Signaling Induces the Transcription of Axin2, a Negative Regulator of the Signaling Pathway. Molecular and Cellular Biology 22, 1172-1183 (2002).

34. Nayak, G. et al. Developmental vascular regression is regulated by a Wnt/betacatenin, MYC and CDKN1A pathway that controls cell proliferation and cell death. Development 145 (2018).

35. Gordon, S.M. et al. The transcription factors T-bet and Eomes control key checkpoints of natural killer cell maturation. Immunity 36, 55-67 (2012).

36. Daussy, C. et al. T-bet and Eomes instruct the development of two distinct natural killer cell lineages in the liver and in the bone marrow. J Exp Med 211, 563-577 (2014).

37. van Helden, M.J. et al. Terminal NK cell maturation is controlled by concerted actions of T-bet and Zeb2 and is essential for melanoma rejection. J Exp Med 212, 2015-2025 (2015).

38. Townsend, M.J. et al. T-bet Regulates the Terminal Maturation and Homeostasis of NK and V. Immunity 20, 477-494 (2004).

39. Robbins, S.H., Tessmer, M.S., Van Kaer, L. \& Brossay, L. Direct effects of T-bet and MHC class I expression, but not STAT1, on peripheral NK cell maturation. Eur J Immunol 35, 757-765 (2005). 
40. Boos, M.D., Yokota, Y., Eberl, G. \& Kee, B.L. Mature natural killer cell and lymphoid tissue-inducing cell development requires Id2-mediated suppression of E protein activity. J Exp Med 204, 1119-1130 (2007).

41. Delconte, R.B. et al. The Helix-Loop-Helix Protein ID2 Governs NK Cell Fate by Tuning Their Sensitivity to Interleukin-15. Immunity 44, 103-115 (2016).

42. Yokota, Y. et al. Development of peripheral lymphoid organs and natural killer cells depends on the helix \pm loop \pm helix inhibitor Id2. Nature 397 (1999).

43. Norrie, I.C., Ohlsson, E., Nielsen, O., Hasemann, M.S. \& Porse, B.T. C/EBPalpha is dispensable for the ontogeny of PD-1+ CD4+ memory T cells but restricts their expansion in an age-dependent manner. PLoS One 9, e84728 (2014).

44. Shimatani, K., Nakashima, Y., Hattori, M., Hamazaki, Y. \& Minato, N. PD-1+ memory phenotype CD4+ T cells expressing C/EBPalpha underlie $T$ cell immunodepression in senescence and leukemia. Proc Natl Acad Sci U S A 106, 15807-15812 (2009).

45. Undi, R.B. et al. Wnt Signaling: Role in Regulation of Haematopoiesis. Indian J Hematol Blood Transfus 32, 123-134 (2016).

46. Hoppler, S. \& Kavanagh, C.L. Wnt signalling: variety at the core. J Cell Sci 120, 385-393 (2007).

47. Lindberg, J., Martin-Fontecha, A. \& Hoglund, P. Natural killing of MHC class Ilymphoblasts by NK cells from long-term bone marrow culture requires effector cell expression of Ly49 receptors. International Immunology 11, 1239-1245 (1999).

48. Lanier, L.L. NK cell recognition. Annu Rev Immunol 23, 225-274 (2005).

49. Assenmacher M1, S.J., Radbruch A. Flow cytometric determination of cytokines in activated murine $\mathrm{T}$ helper lymphocytes: expression of interleukin-10 in interferon-gamma and in interleukin-4-expressing cells. Eur J Immunol. 24, 10971101 (1994). 


\section{Millan Figure 1}

A.

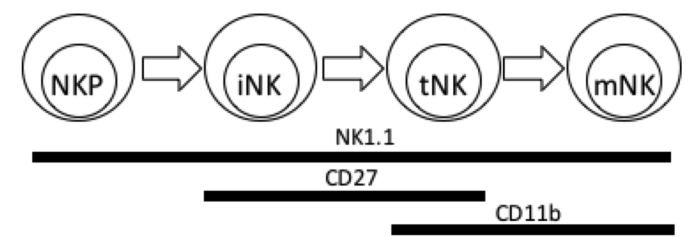

D.

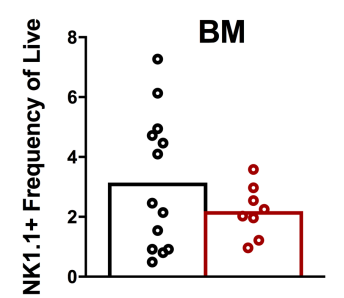

E.

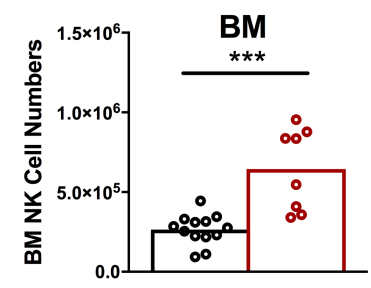

B.

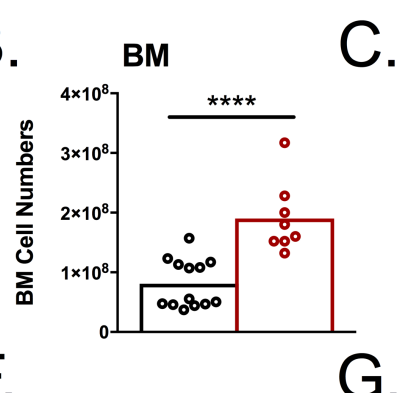

F.

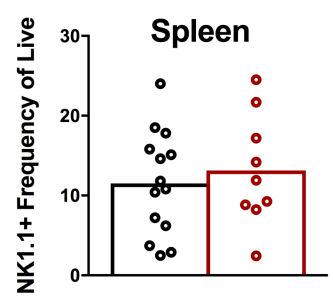

$\mathrm{G}$.
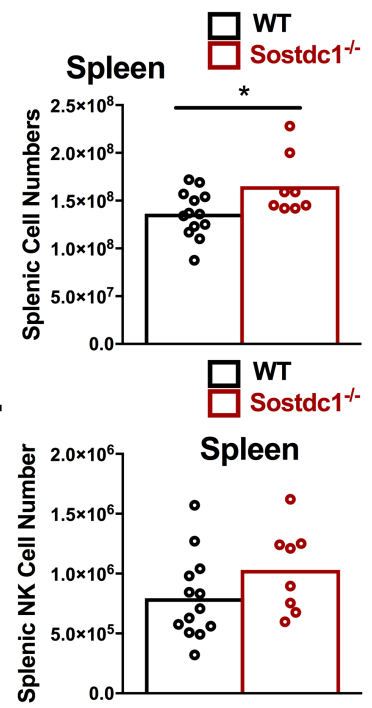

$\mathrm{H}$.

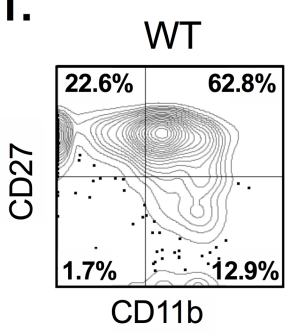

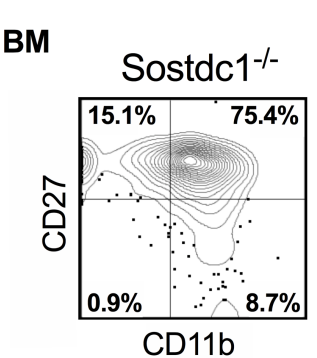
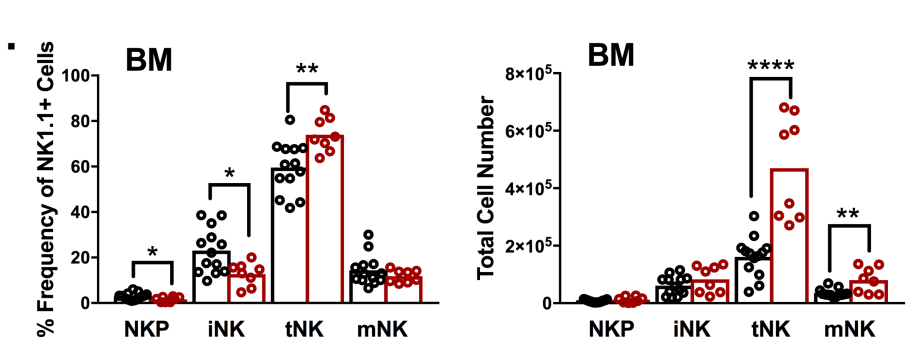

J.

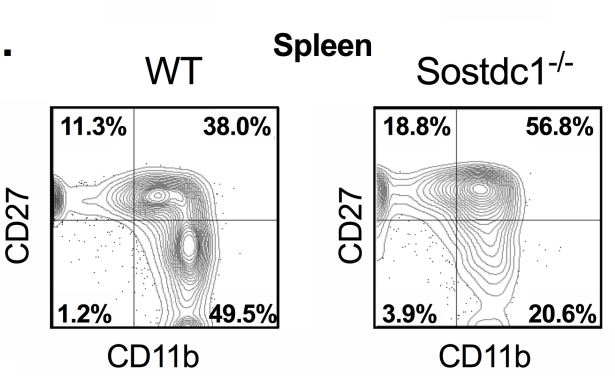

$\mathrm{K}$.
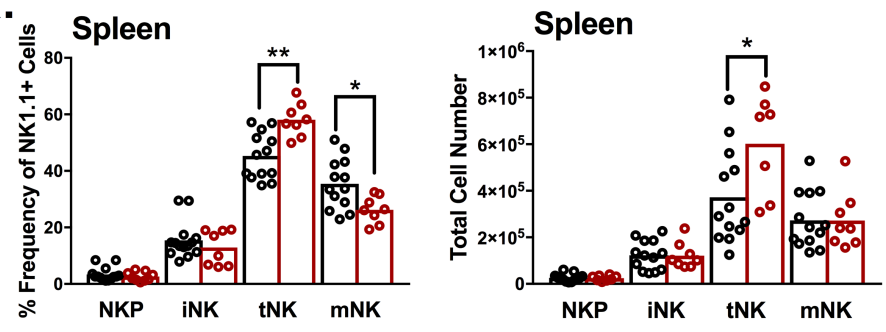

L.

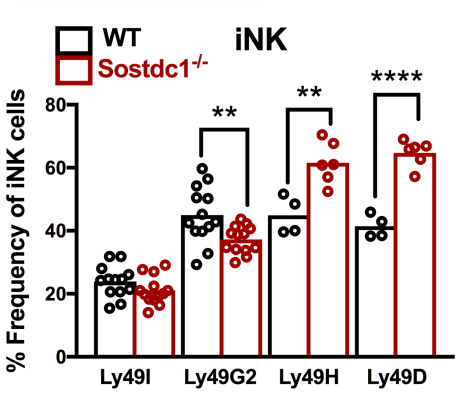

M.

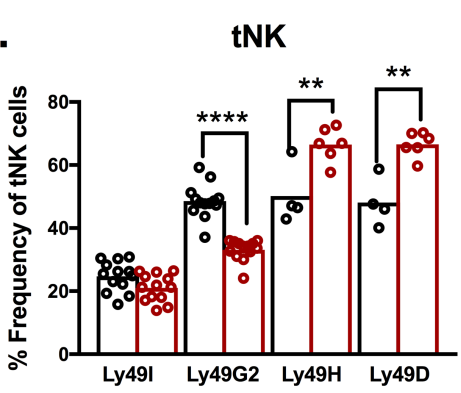

$\mathrm{N}$.

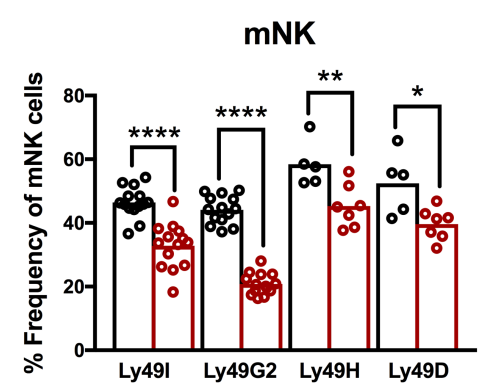


bioRxiv preprint dol: https://doi.org/10.1101/387225; this version posted August 9, 2018. The copyright holder for this preprint (which was not certified by peer review) is the author/funder. All rights reserved. No reuse allowed without permission.

Milan Figure 2

A.

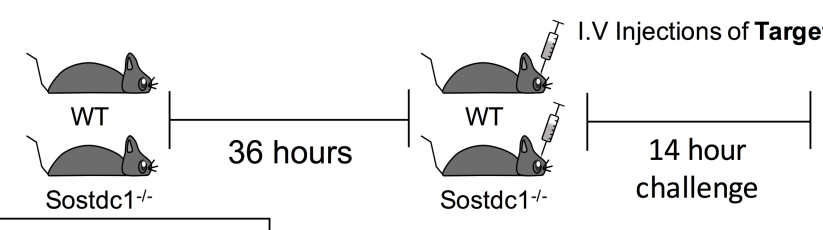

I.P Injection of Poly (I:C)

C.

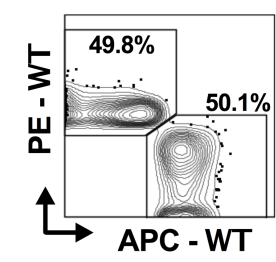

D.

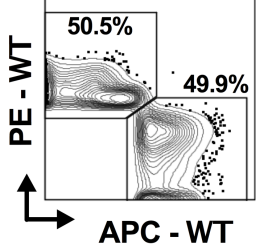

WT Host
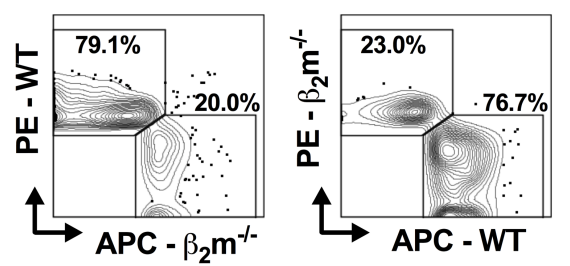

Sostdc1 $1^{-/-}$Host
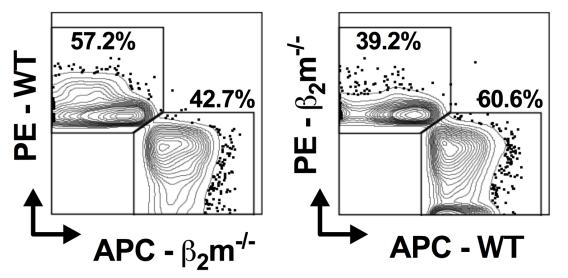

B.

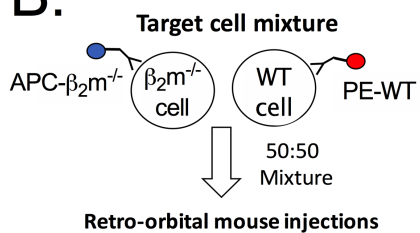

E.

Mouse recipients

WT

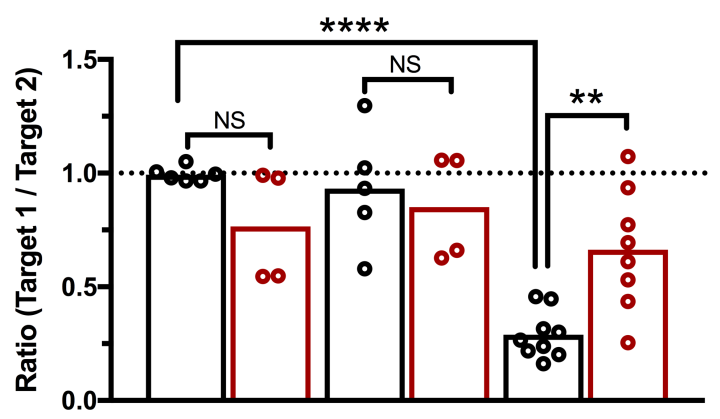

\begin{tabular}{|l|c|c|c|}
\hline Target 1 & WT & Sostdc1-- $^{--}$ & $\beta_{2} \mathrm{~m}^{--}$ \\
\hline Target 2 & WT & WT & WT \\
\hline
\end{tabular}




\section{Millan Figure 3}

A.

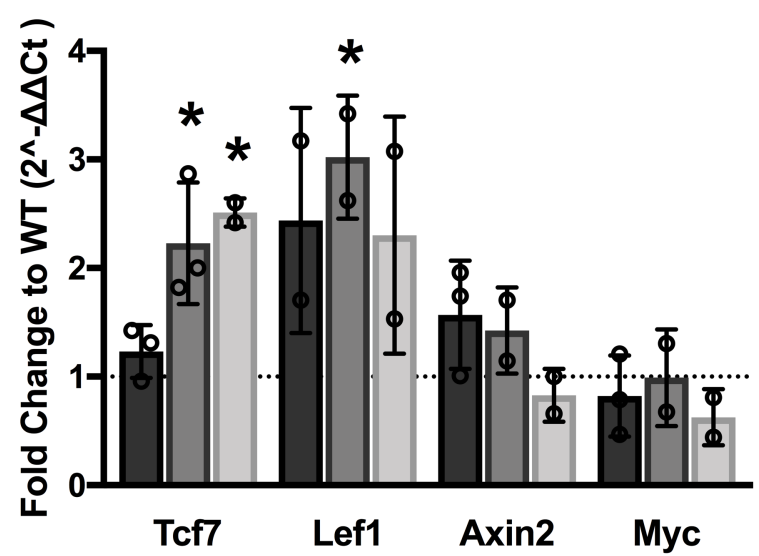

B.

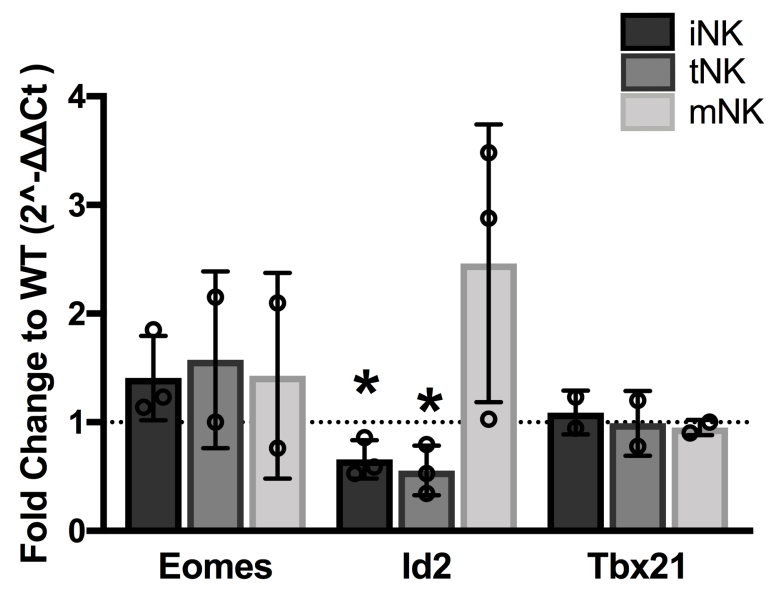




\section{Millan Figure 4}

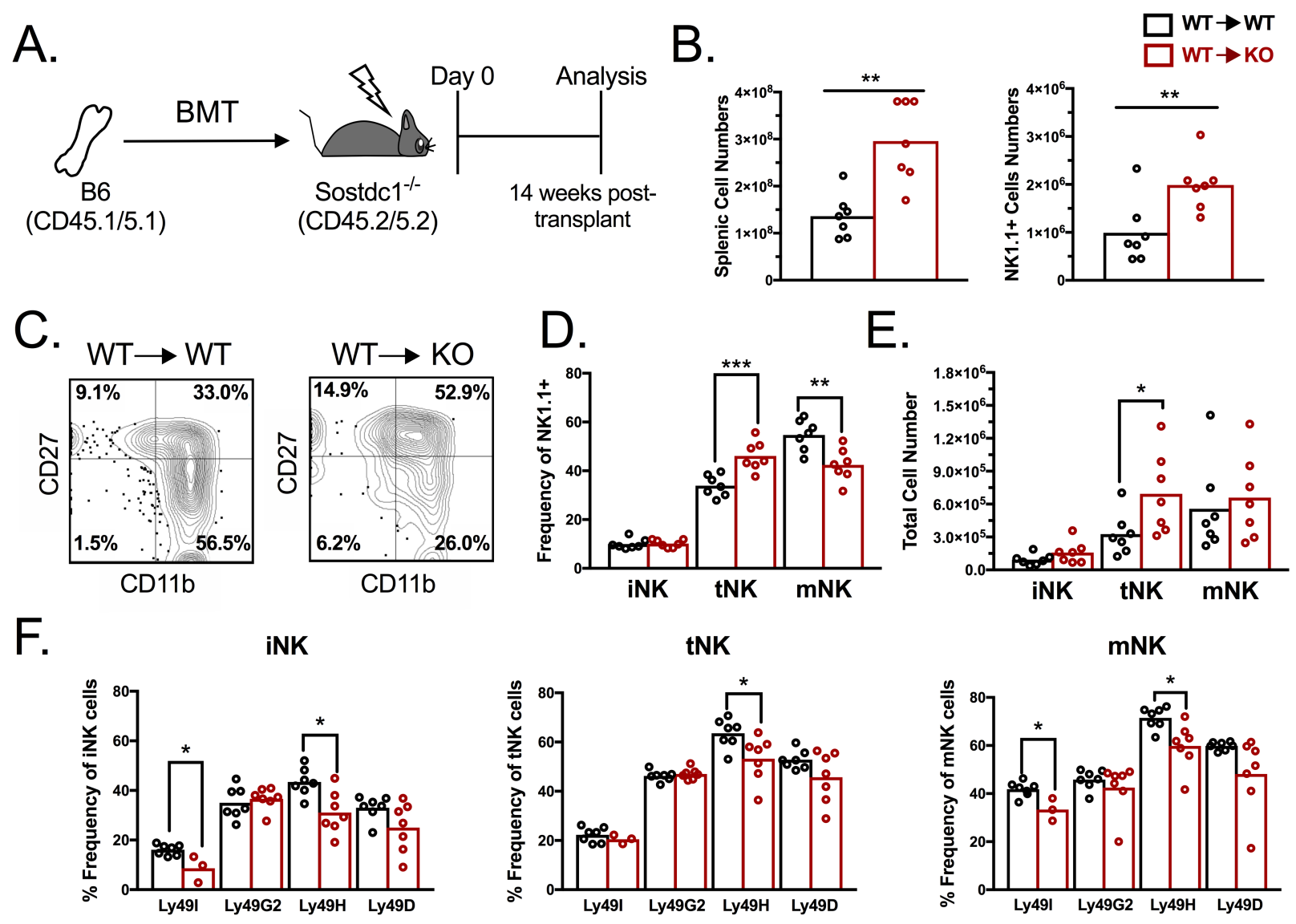




\section{Millan Figure 5}

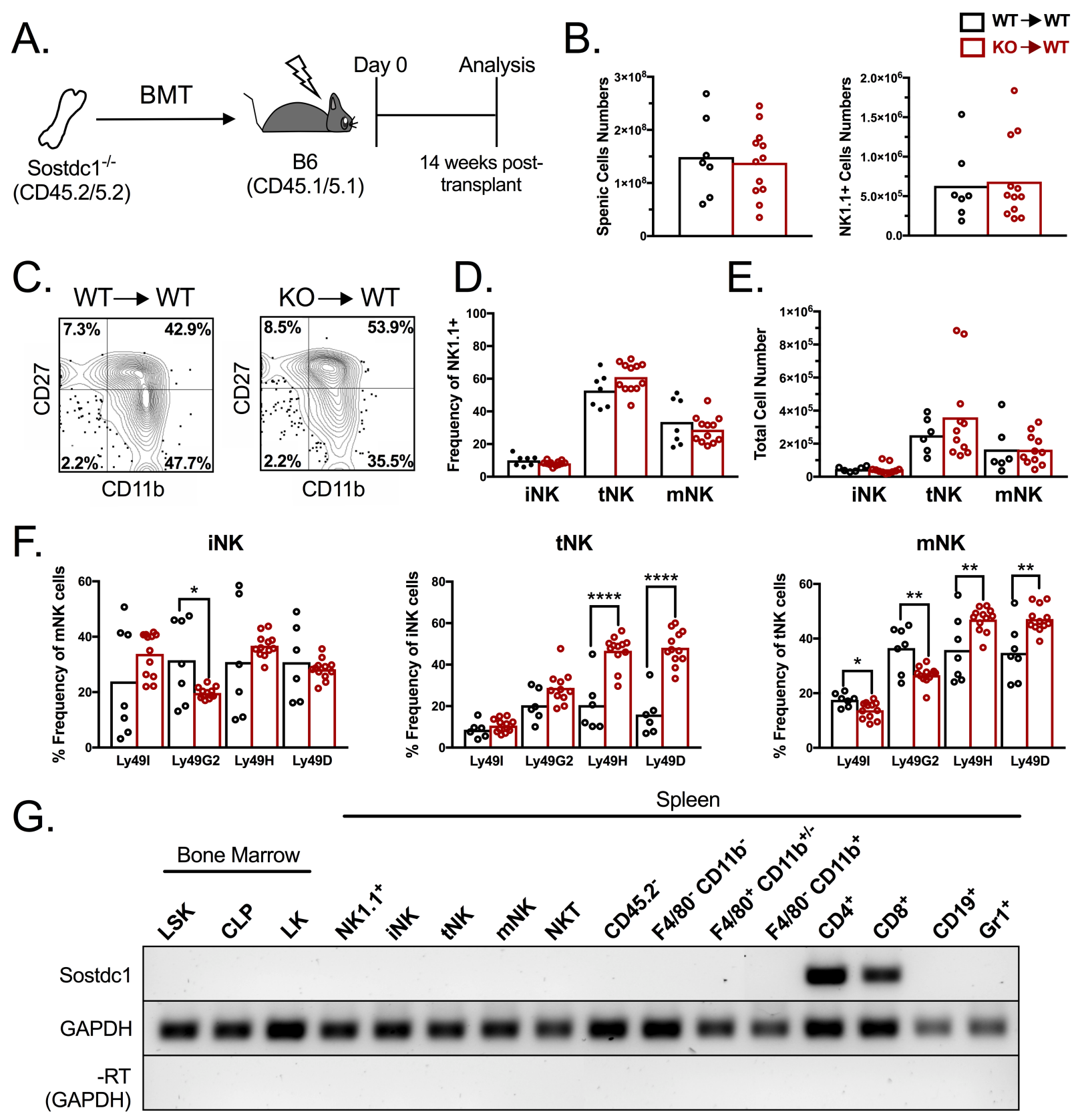




\section{Millan Figure 6}

A.

\section{Sostdc1-Sufficient Microenvironment}

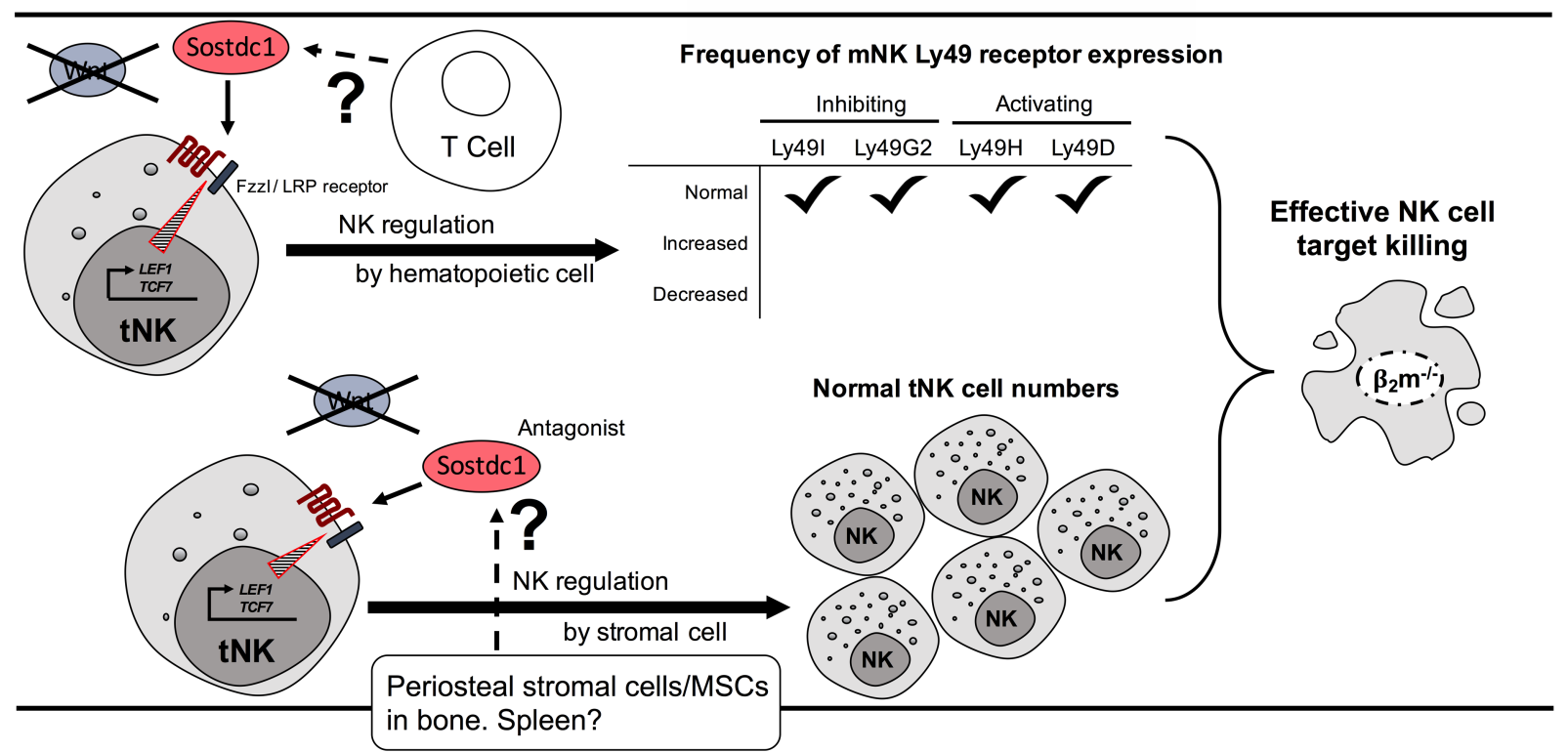

B.

\section{Sostdc1-Deficient Microenvironment}

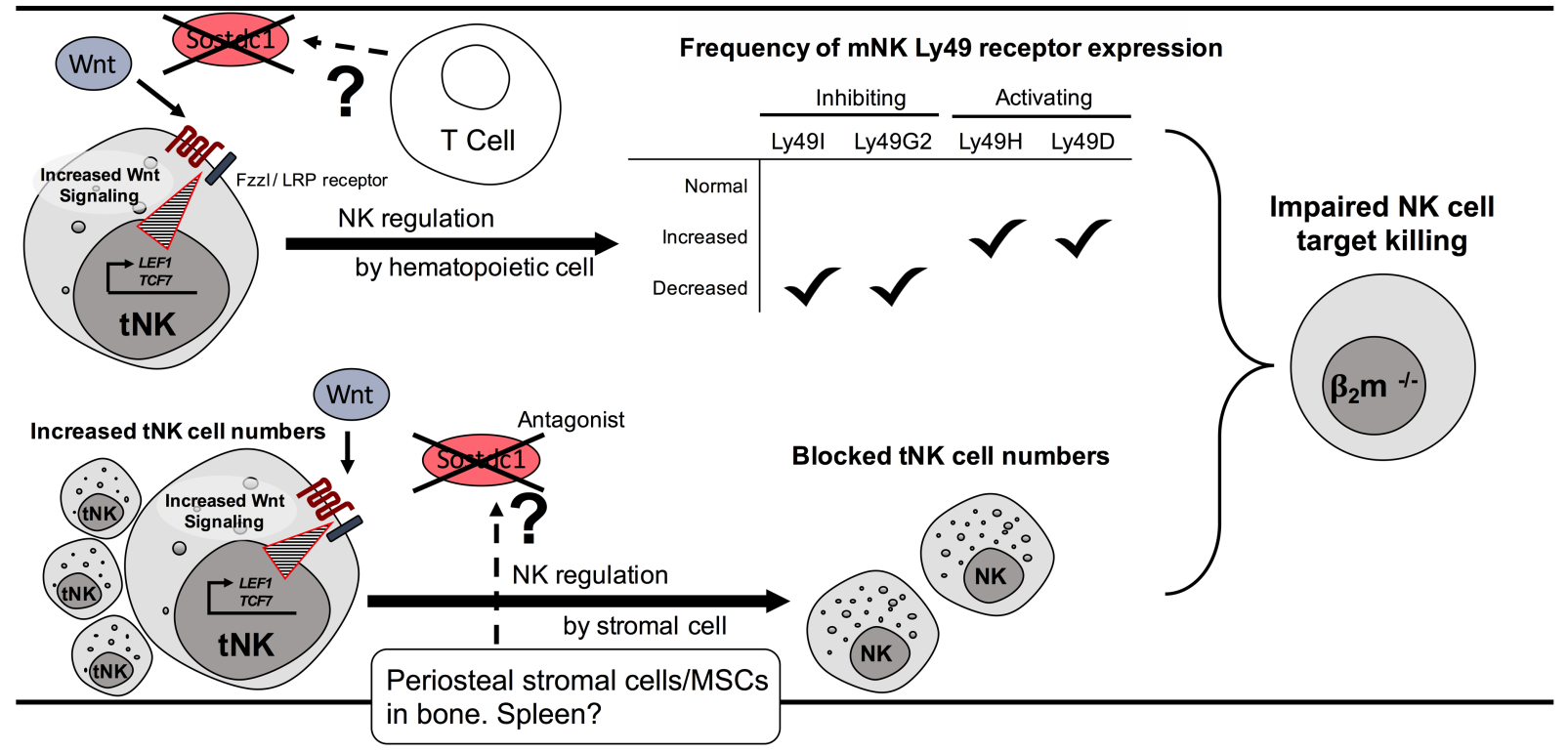

